\title{
Implementation of a Security System, Using Captured Fingervein, Applying the Concepts of Machine Learning
}

\author{
${ }^{1}$ Oyinloye Oghenerukevwe Elohor ${ }^{*},{ }^{2}$ Akinbohun Folake, ${ }^{3}$ Thompson Aderonke, ${ }^{4}$ Korede Bashir \\ ${ }^{1,4}$ Department of Computer Science, Ekiti State University Ado-Ekiti, Ekiti State, Nigeria \\ ${ }^{2}$ Rufus Giwa Polythenic, Owo, Ondo State, Nigeria \\ ${ }^{3}$ Federal University of Technology Akure, Ondo State, Nigeria
}

\begin{abstract}
:
This work explores the field of biometric finger vein recognition - which is the identification of individuals using the unique vein patterns under their finger skins. This work also includes the implementation of an Android fingerprint biometric system using the Android Near InfraRed (NIR) module, which exists to show the similarities and differences between the two (fingervein and fingerprint) prevalent biometric features. This work thus confirms that finger vein recognition shows great promise as an accurate solution to modern society's problem of automated personal authentication
\end{abstract}

Keywords: Biometric, Fingervein, Machine Learning, Security, Token

\section{Introduction}

Finger vein recognition is a promising biometric recognition technology which verifies identities through finger vein patterns. Medical studies have shown that the finger vein pattern is unique and stable. In detail, the finger veins of an individual are different from the others', and even the veins captured from a single individual are quite different from one finger to another. Furthermore, the finger veins are also invariant for healthy adults.

Compared with fingerprints, finger veins are hard to be forged or stolen as they are hidden inside the fingers. The contactless captures of finger veins also ensure both convenience and cleanliness, and they are user-friendly. Furthermore, Finger veins are less affected by physiology and environment factors such as dry skin and dirt.

A typical finger vein recognition process is composed of the following four steps. Firstly, the finger vein images are obtained via the finger vein capturing devices. Secondly, the finger vein images are preprocessed. Thirdly, the features are extracted. Finally, the finger vein images are matched based on the extracted features.
The preprocessing procedure includes image enhancement, normalization, and segmentation. For image enhancement, Yang and Yan incorporated directional decomposition and Frangi filtering to enhance the image quality. Yu et al. proposed an enhancement algorithm based on multi-threshold combination. Yang and Yang introduced multichannel Gabor filter to enhance the images and obtained better performance. Finger vein segmentation is also a very important step, and there are some typical methods including line tracking, mean curvatures, and region growth-based feature. A detailed description of these approaches is beyond the scope of this report.

Principal component analysis (PCA) is a popular linear dimensionality reduction and feature extraction technology. It has extensive applications in image processing. Wu and Liu extracted the PCA features and then trained a neural network for matching, which results in a high recognition rate. Since PCA transforms the 2-dimensional image matrix to a 1-dimensional vector, the covariance matrix is always large in size and it is time intensive to obtain the projection matrix which is composed of the covariance matrix's eigenvectors. Yang et al. proposed 2D PCA to reduce the size of the 
covariance matrix and save time for computing projection matrices. In order to represent the characteristics of the 2-dimensional images more accurately, Zhang and Zhou introduced (2D)2 PCA which can reflect the information of the image in row and column directions, respectively, use less time to compute the projection matrix, and get better experimental results on face recognition.

Recently, more and more researchers apply machine learning methods to finger vein recognition. Liu et al. introduced manifold learning to finger vein recognition. $\mathrm{Wu}$ and Liu used PCA and LDA to extract features and train an SVM model for recognition. Measuring the distance of the two samples is the premise of machine learning. For example, $\mathrm{KNN}$ requires a distance metric to find the neighbours of the target instance and then conducts classification or regression based on the distance metric. Typical distance metrics, such as Euclidean distance, make significant contribution in some application domains. In some conditions, these metrics cannot satisfy the assumption that the distances between instances from the same class are small while those from different classes are large. It limits the utilities of most machine learning methods.

\section{A. Problem Statement}

There are two challenges for finger vein recognition:

I. how to efficiently extract distinguishing features and

II. how to design a strong classifier with high recognition rate and fast recognition speed to make the system more practical in real-world applications.

To overcome the stated challenges in the problem statement, in this project, the (2D)2 PCA method is applied to extract the features from finger vein images. In order to address the shortcoming of traditional distance-metric-based classifiers, a classifier is built for each individual based on metric learning.

\section{B. Specific Objectives}

The specific objectives of this paper are to:

I. Analyse current literatures on existing vein recognition methods.
II. To design a finger vein detection system using clustering techniques.

III. To implement the finger vein detection system as a security device

IV. To evaluate the performance of (II) and (III).

\section{Methodology}

A research of biometric vein detection technique was carried out. The methodology of this project is to break down the finger vein recognition problem into the three stages of, "Image pre-processing", "Feature extraction" and "Classification", and develop each of these stages individually before combining them to form a complete biometric system.

The proposed method includes training process and recognition process. A classifier is built for each individual, and the samples from a certain individual are treated as positive and others are negative. In the verification mode, we input a test sample to corresponding classifier to verify whether the sample comes from this individual based on the classification result. In the identification mode, we input a test sample to every classifier and identify which individual this sample belongs to. The performance of the biometric system is used in evaluating the stated methodology utilized in this project which is given by the set of equation as shown in Equations 1.1 and 1.2.

$$
\begin{gathered}
F A R=\frac{\text { number of imposters accepted by the system }}{\text { total number of imposter access attempts }} \\
F R R=\frac{\text { number of genuine users rejected by the system }}{\text { total number of genuine user access attempts }}
\end{gathered}
$$

\section{Literature Review}

Like fingerprints or iris patterns, fingervein-based blood vessel patterns are unique for each individual. Fingervein-based blood vessel pattern have high security because the veins are located under the surface of the skin. The fingerprints can be cheated by dummy finger fitted with a copied fingerprint, but the fingervein-based identification system is highly secure for authentication. 
For authentication application the pattern of the finger vein is stored in a database. The finger is placed on an attester terminal which contains a near-infrared, light emitting diode light source and a monochrome charge coupled device camera.

The hemoglobin present in the blood absorbs the near infrared light emitting diode light and makes the vein to appear as dark pattern. The recorded image is digitized and stored in the database. During authentication, the finger vein is scanned and is compared with the image in the database.

In the following section, we review the related works on finger vein biometric security system over various applications.

David et al [1] introduced preliminary process to enhance the image quality that worsen by light effect and produces noise by the web camera, then segmented the vein pattern by using adaptive threshold method and matched them using improved template matching. The result shows that even the image quality is not good and as long as the veins are clear with some appropriate process can be used for personal identification.

Wenming et al [2] proposed a structured personal identification approach using finger vein Location and Direction Coding (LDC). Initially finger vein imaging device is designed using Near-InfraRed (NIR) light source, by which a database for finger vein images is established. The brightness difference in the finger vein image is used to extract the vein pattern. Then finger vein LDC creates a structured feature image for each finger vein. The structured feature image is utilized to conduct the personal identification with image database for finger vein, which includes 440 vein images from 220 different fingers.

Hua-Bin et al [3] presented an algorithm based on adaptive filtering and retinex method for enhancement of hand vein images. The principal of the near-infrared hand vein image acquisition is introduced, then the retinex method is used to normalize hand vein images, and the adaptive smoothing method is selected to estimate the illumination. Then the gray cosine transform is used to enhance the discrimination of the skin and the vein in hand vein images. Then a determination criterion of hand vein is established to remove the false vein blocks from the segmented hand vein images.

Shi et al [4] proposed a method to make low cost devices using vein pattern images with low contrast, and high-quality images. The method could extract the vein network successfully as using high-quality images. The principle of vein imaging is discussed to acquire the vein images which could enhance the contrast and the algorithm of extracting the vein pattern from low quality images.

Desong et al [5] presented a more secure and reliable user identification mechanism using biometrics technology equipped into the consumer electronics devices. The system uses finger-vein identification system which provides high security and reliability than other identification technology. The algorithm composes of a feature extraction using radon transform and singular value decomposition and classification using a normalized distance measure.

Zhi et al [6] proposed a real-time embedded fingervein recognition system for authentication on mobile devices. The system is implemented on a DSP platform and equipped with a novel fingervein recognition algorithm. The system takes about 0.8 seconds to verify one input finger-vein sample and achieves an equal error rate of 0.07 percent on a database of 100 subjects. The results proved that the finger-vein recognition system is qualified for authentication on mobile devices.

Li et al [7] proposed a modality-based bi-finger vein verification system. Both the finger vein and finger shape could be extracted from the single image acquired from the sensor. The system includes the new finger vein network extraction algorithm. The intersection of the forefinger and middle finger as the origin is introduced to the coordinate system, proposing a method including determining the region of interest, the finger vein and shape features extraction and its corresponding fusion verification.

Lin et al [8] presented an algorithm for segmenting the dorsal hand vein image and extracting the vein skeleton. After gray and size normalizing, Gaussian low pass filter and median filter are used to eliminate the speck noise and the horizontal strip 
scanning noise respectively. Then an improved NiBlack algorithm segments the vein pattern and an area thresholding algorithm removes the noise blocks from the vein pattern.

Jinfeng et al [9] focused on finger-vein enhancement and segmentation based on Gabor filters in the spatial domain. Considering the high randomicity of the finger-vein networks, a bank of even symmetric Gabor filters with eight orientations is firstly used to exploit vein information in images. Then, image reconstruction is implemented to generate an image containing an integrated fingervein network.

Gongping et al [10] proposed a finger vein recognition method based on a Personalized Best Bit Map (PBBM). The method is rooted in a local binary pattern-based method and then inclined to use the best bits only for matching. The recognition framework consists of preprocessing, feature extraction, and matching. For evaluating the effectiveness of the method extensive experimental designs are made and results show that PBBM achieves better performance.

Naoto et al [11] proposed a method of personal identification based on finger vein patterns. An image of a finger captured under infrared light contains not only the vein pattern but also irregular shading produced by the various thicknesses of the finger bones and muscles. The method extracts the finger vein pattern from the unclear image by using line tracking that starts from various positions.

Lee et al [12] utilized static features together with dynamic features for fingerprint vitality detection. Before this method was proposed, the static and dynamic features of a fingerprint had been studied separately. The authors studied fingerprint silicon replicas by designing a vitality detection system using an optical capture device to prevent spoof attacks from gelatin or silicon fake-fingerprints from deceiving some commonly used fingerprint sensors.

Hashimoto et al [13] applied the original concept of fuzzy vault to the fingerprint minutia data. In this method, a 128-bit cryptographic key is feasibly bound with the fingerprint minutia data, but this method requires image alignment. Fingerprint minutia data was protected by designing a fuzzy vault for fingerprint data.

$\mathrm{Wu}$ et al [14] proposed a system that provides for segmentation and enhancement of latent fingerprints building on the significant progress that have been made in improving the matching accuracy of latent fingerprints. Latent segmentation and enhancement algorithm were proposed to refine a poor fingerprint image. By using a total variation decomposition model, the piecewise-smooth background noise can be removed and several overlapping patches are defined and used for latent enhancement, leading to better matching performance.

Flynn et al [15] utilized the fuzzy commitment scheme to protect fingerprint features. Since it is convenient to have biometric features in the binary format, the authors processed the features with a randomized dynamic quantization transformation. Fuzzy commitment scheme was used to design a biometric template for protecting fingerprint features.

\section{Methodology}

The process of biometric pattern recognition is, as described in Chapter 1, split into a common pipeline of three stages: pre-processing, feature extraction and classification. The methodology of this study is to break down the finger vein recognition problem into these three stages, and develop each of these stages individually before combining them to form a complete biometric system.

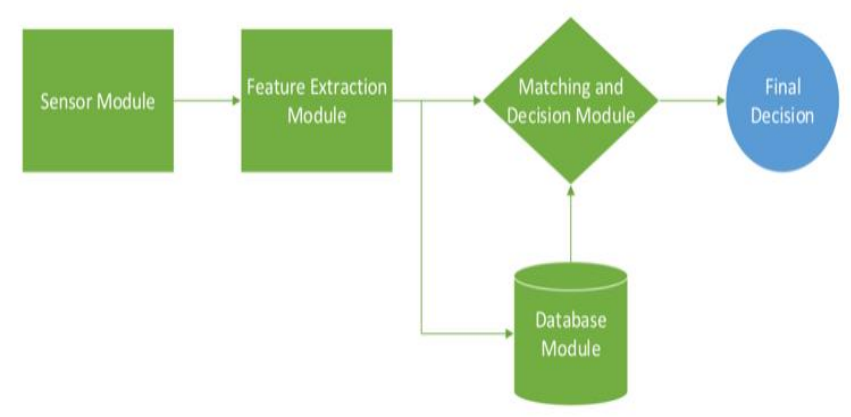

\section{Figure 1.1: General architecture of a biometric identification system}

1) Sensor module: The scanner used to obtain raw biometric data from the user. In the case of vein recognition, it consists of an infrared 
(IR) camera and an IR illumination source (such as a bank of IR LEDs).

2) Feature Extraction module: This module extracts features which accurately represents the raw biometric data. During the enrolment phase, these features are stored in the database and are known as templates or feature vectors.

3) Database module: This acts as a repository for all biometric information required by the system to make future decisions. Security measures are also required so that the feature vectors used for identification are not stolen by attackers.

4) Matching and decision module: Compares extracted features against the stored templates to generate matching scores. If the score is below a certain threshold, the query image is matched to the stored template. Otherwise, it is rejected.

In this study, stored images refer to raw images used to populate the database whilst query images refer to images acquired during the authentication process.

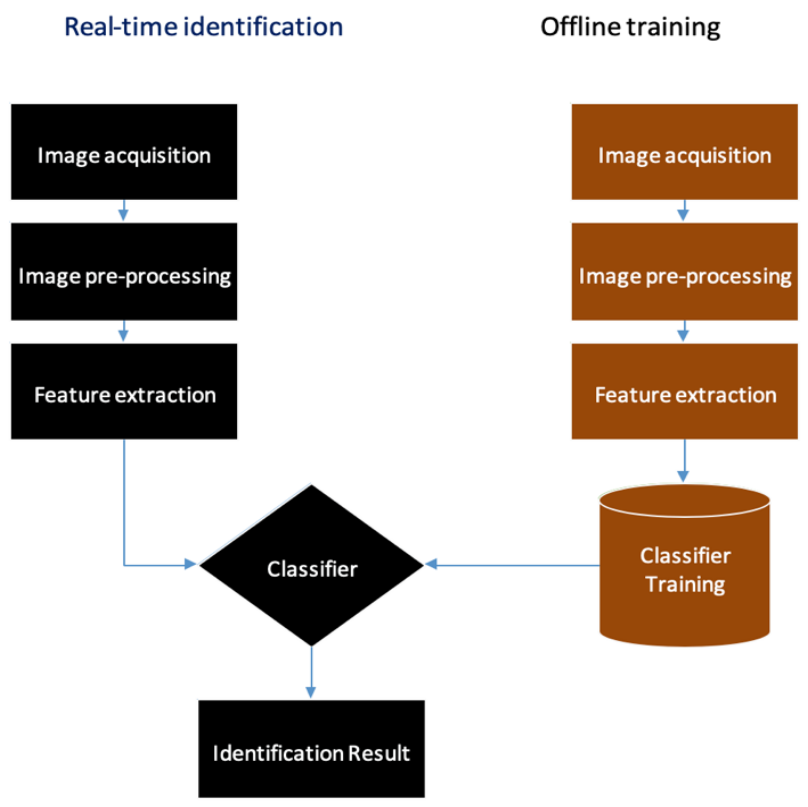

Figure 1.2: Architecture of the proposed biometric systems (grouped into offline training mode and real-time verification mode)

\section{A. Image Processing}

Image processing refers to signal processing operations which are performed on images (Acharya et. al, 2005). It is used extensively in the pre-processing and feature extraction stages of the biometric pipeline. As a result, contrast enhancement techniques are discussed, mathematical morphology and segmentation likewise, which all have relevance to vein recognition.

1) Contrast Enhancement: Contrast enhancement is relevant since features are easier to extract from images with greater contrast. Moreover, vein images tend to have non-uniform illumination and low contrast. This is not only the fault of the imaging hardware, but also due to the fact that the human finger scatters the Near InfraRed (NIR) light, penetrating it similar to the way that visible light is scattered by fog.

2) Histogram Normalization: Histogram normalisation is a simple contrast enhancement method which "stretches" the dynamic range of pixel intensity values by linearly scaling the intensities such that light pixels become even lighter and dark pixels even darker. This point-wise operation is computed by:

$$
I^{\prime}(x, y)=\frac{R_{\max }-R_{\min }}{I_{\max }-I_{\min }} \times\left(I(x, y)-I_{\min }\right) \forall x, y \in I
$$

where Rmax and Rmin represent the new maximum and minimum gray levels respectively, I is the original image and I'is the output image.

3) Adaptive Histogram Equalisation: Global histogram equalisation techniques, however, do not take local details into account and can end up magnifying noise. To overcome this, histogram equalisation can be performed locally, by sliding a twodimensional window over the image and performing equalisation separately on each region falling within the window. A popular algorithm which implements this is CLAHE - Contrast Limited Adaptive Histogram 
Equalisation - and is available in the MATLAB Image Processing Toolbox.

4) The Optimal Contrast Enhancement Technique: Olsen et al. conducted a comparison of contrast enhancement techniques for vein recognition due to its importance in the pre-processing stage. CLAHE was found to outperform the normalisation technique proposed by Wang. However, the optimal algorithm was STRESS (Spatio-Temporal Retinex-like Envelope with Stochastic Sampling) which is an algorithm that is not considered in this study due to its complexity.

5) Mathematical Morphology: This is the analysis of signals in terms of shape and was pioneered by Serra. It treats both images and shapes as a set of points and processes them accordingly using local transformations (Olsen et al, 2005). The discussion here will only consider binary images, which the field was originally developed for.

6) Erosion and Dilation: The simplest morphological operations are erosion and dilation. The erosion operator reduces the image $X$ as it requires that all points in the structuring element $\mathrm{B} 1$ be contained in $\mathrm{X}$. This is shown as:

$$
X \odot B=\left\{x \mid B_{x}^{1} \subset X\right\}
$$

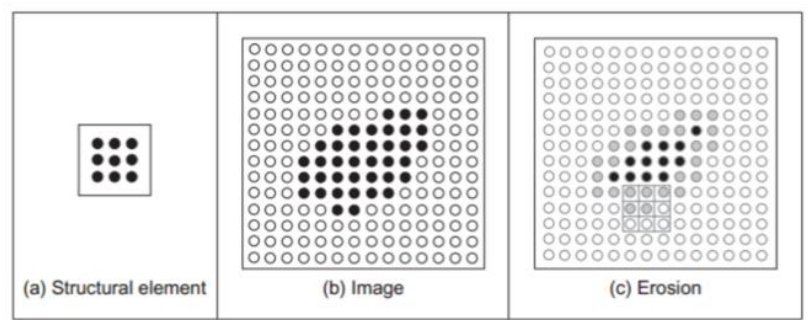

Figure 1.3: An erosion operator. As it can be seen, the boundaries of the binary image are reduced.

7) Applications of Morphology: A relevant application of morphology to vein recognition is thinning. As the diameter of an individual's veins can change due to environmental conditions, it is important to thin veins to a width of one pixel such that our description of the vein is invariant to changes in vein diameter.

Thinning can be achieved by iteratively eroding an object until it has been reduced to a line a single pixel wide. Two well-known algorithms which achieve this operation are the Stentiford Thinning Algorithm and the Zhang-Suen Thinning Algorithm. The latter is implemented in the MATLAB Image Processing Toolbox.

8) Segmentation: Segmentation involves partitioning an image into regions which share common attributes or properties. In the context of vein recognition, an area of interest would be in segmenting the veins from the rest of the image. Common techniques such as global thresholding using Otsu's method and NiBlack local thresholding are not effective due to nonuniform illumination.

Several algorithms, however, have been developed to extract vascular patterns from images. The most notable are Miura's "Repeated Line Tracking" algorithm, Miura's "Maximum Curvature" method and Huang's "Wide Line Detector".

9) Feature Extraction: In Computer Vision, a feature refers to "interest points" on an image (Zhang et al, 2011). It captures the most important information in an image, and hence can be considered to be a form of dimensionality reduction. When the data input to a pattern recognition system is too large to be processed, it makes sense to extract the key features and process those.

In addition to dimensionality reduction, a more robust method of comparing two images is to compare their features rather than their individual pixels. This is because changes in image size, translation or rotation can cause a pixel-wise comparison to fail. On the other hand, features which possess geometric invariance will not be affected at all (Zhang et al, 2011). 


\section{Test and Result}

Building on the methodology expounded in the last chapter, in this chapter, two biometric systems will be designed which use finger vein minutiae as features to identify individuals. In keeping with the system architecture described in figure 1.1, the design of the pre-processing, feature extraction and classification stages are detailed in order. The preprocessing stage is common to both systems and is discussed next in the following section.

The vein imaging device used in capturing the images for the dataset used in this project was specifically designed to deter the subject from rotating his finger during capture.

\section{B. Pre-processing}

In order to extract the intersection points of veins, there is a need to first segment the veins from the rest of the image. The goal of this stage is to produce a binarised image where the foreground (white) pixels represent the actual vein and the background (black) pixels represent the remaining tissues of the finger.

The extraction of the Region of Interest (ROI) and subsequent contrast enhancement using the CLAHE algorithm were the first steps completed and are detailed. Thereafter, the algorithms examined to segment the veins from the images.

1) Region of Interest (ROI) Extraction: The images from the SDUMLA-HMT database have dimensions of $320 \times 240$ pixels. The images have an unwanted background and the boundary between the finger and scanning lens is also disproportionately bright. The ROI was thus defined as a $320 \times 80$-pixel rectangle representing the middle part of the finger, also shown in Figure 1.4.

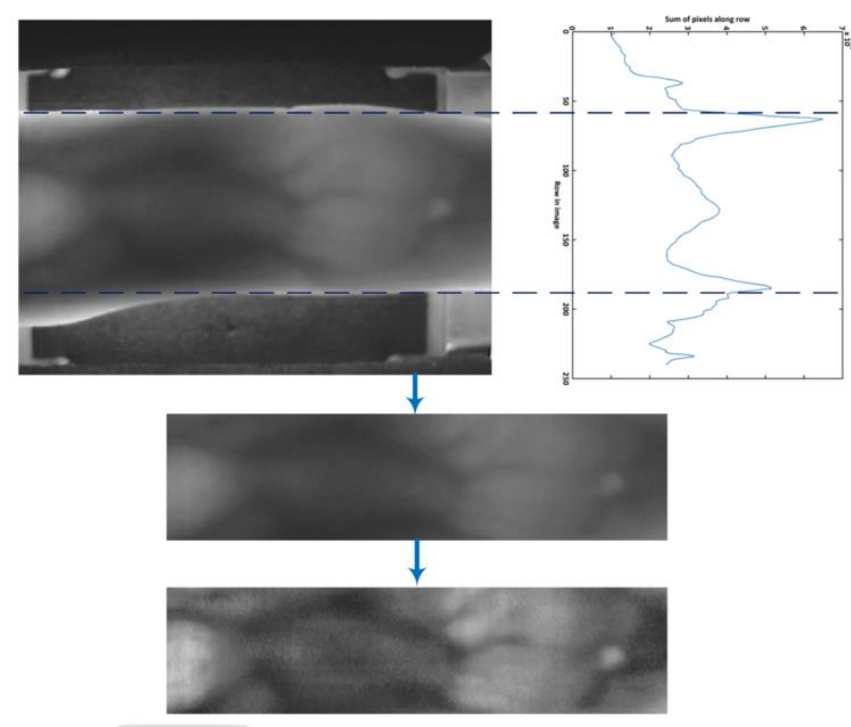

Figure 1.4: Flow diagram of pre-processing. Top: The original vein image and corresponding illumination profile. Middle: The ROI extracted from the image. Bottom: Contrast improvement using CLAHE algorithm produces a more defined image.

This region was obtained by taking the illumination profile along the vertical axis (by summing all the pixels along the rows of the grayscale image) and retaining pixels along the middle 80 rows from the peaks of the profile. These peaks correspond to the boundary of the finger and scanning lens, which contains the brightest pixels (since brighter pixels have higher intensity values than darker ones). In general, the observed finger region is approximately 100 pixels wide. However, a conservative estimate of 80 pixels was used to account for variation in finger widths. Finally, the contrast of the image was improved using the CLAHE algorithm results in the darker veins becoming more distinguishable.

2) Vein Segmentation: The final, and most important stage of pre-processing is to extract the veins and create a binary, thresholded. Common thresholding techniques do not work due to the non-uniform illumination in vein images. 


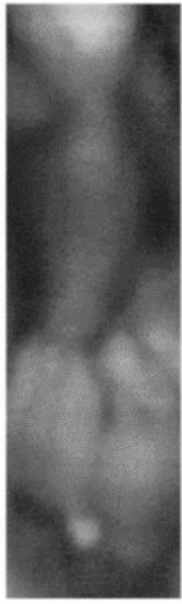

(a) Original image

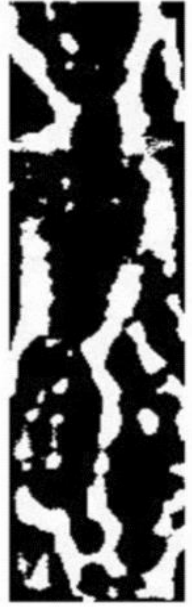

(b) Author's method
Figure 1.5: Segmentation of fingervein image using our segmentation algorithm

\section{Feature Extraction}

Figures and tables must be centered in the column. Large The output of the pre-processing stage is a binary image where the white, foreground pixels represent the actual vein. In this section, the positions of bifurcation points are extracted as our first set of features. Bifurcations are the points where one vein splits into two. Contrastingly, it can also be thought of as the points where two veins intersect. Bifurcations are extensively considered to be reliable features, since the number and position of these points are unique among individuals.

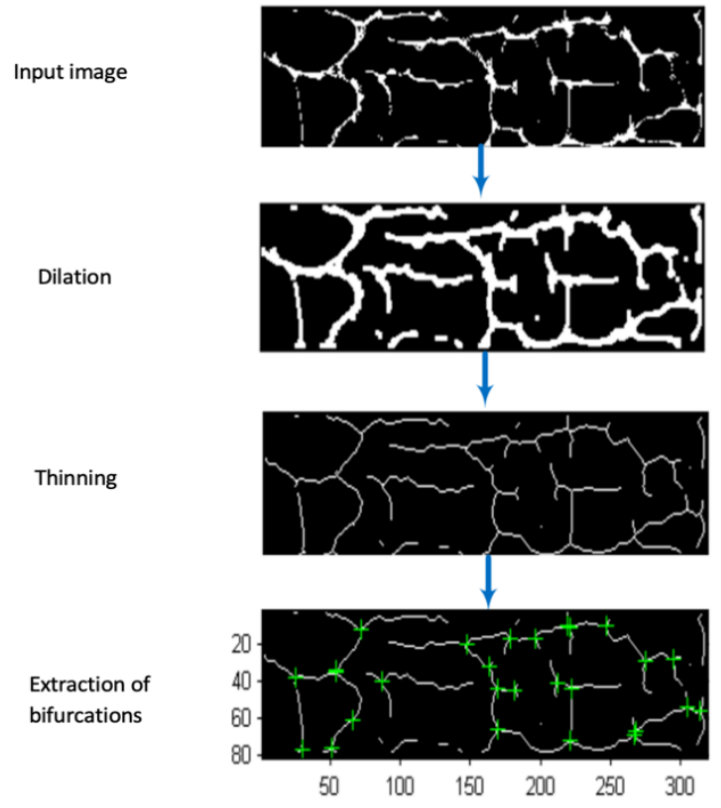

Figure 1.6: Flow diagram of the feature extraction process. The image is first dilated and thinned
Before the bifurcations, which are shown in green,are extracted using a "hit-and-miss" operation

1) Dilation and Thinning: As seen in Figure 4.5(b), the veins thresholded from the input image contain minor discontinuities. This can lead to bifurcations being extracted incorrectly. To combat this, the image was first dilated. It was observed empirically that an appropriate structuring element for the operation was a $3 \times 3$ matrix of unit value. Dilation has the adverse effect of thickening the veins. However, this was irrelevant as the binary image is thinned immediately afterwards using the Zhang-Suen thinning algorithm. Bifurcations can then be extracted from the thinned image. Furthermore, an additional benefit of thinning is that it ensures that the extracted features are invariant to the diameter of the subject's veins which are known to change due to environmental conditions and over a person's lifetime.

The importance of dilating the veins before thinning them is shown by Figure 1.6. The result is much noisier when prior dilation is not performed due to the discontinuities between the original segmented veins. Furthermore, this can cause false bifurcations to be extracted.

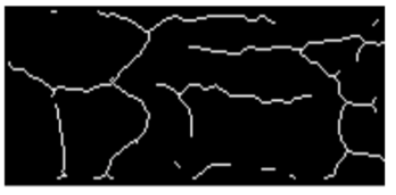

(a) Dilation before thinning

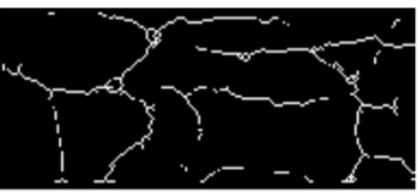

(b) No dilation before thinning
Figure 1.7: Not dilating before thinning results in a noisy output which can give rise to false bifurcation points

2) Bifurcations: A pixel is defined as a bifurcation point if it is a white pixel (part of the vein), and its eight neighbouring pixels lying within a $3 \times 3$ region transition from white-to-black or black-to-white six or more times in a clockwise direction. These points were extracted using the morphological "hitand-miss" operation and employed three structuring elements in as well as all seven possible $45^{\circ}$ rotations of them. 
Once the bifurcations have been obtained, our features can be thought of as a list of $x$ and $y$ co- ordinates which represent the bifurcation points on the veins. It was observed that there are between 20 and 50 bifurcations on each sample. These features can be visualised graphically by the scatter plots, Figures 4.6 and 4.7 , which show the $\mathrm{x}$ and $\mathrm{y}$ co-ordinates of the bifurcations.

It can be seen that the bifurcation positions in the first scatter plot, Figure 1.8, are very similar to each other whilst it is also evident that the positions vary greatly in Figure 1.9. In Figure 1.8, the bifurcations are not exactly identical even though they are from the same individual. Moreover, the total number of bifurcations in the two images are different. This difference is due to small variations in the two sample images as well as the fact that the vein extraction algorithm used can make segmentation errors when faced with non-uniform illumination as discussed. It can also be seen from Figure 1.8 that although most of the points are closely correlated, there are a few outlier samples which do not match each other.

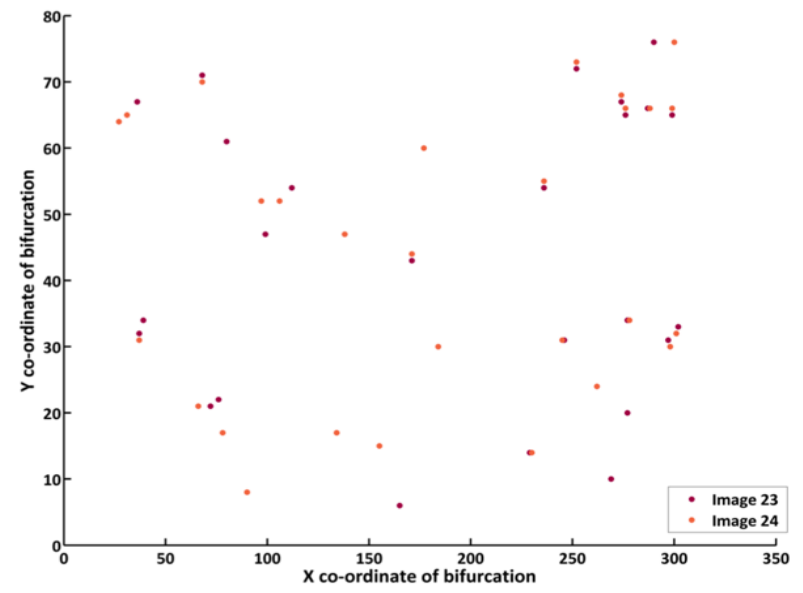

Figure 1.8: A scatter plot of the positions of the bifurcations of two images of the same class. Note that some of the bifurcations are so close to each other they appear as one.

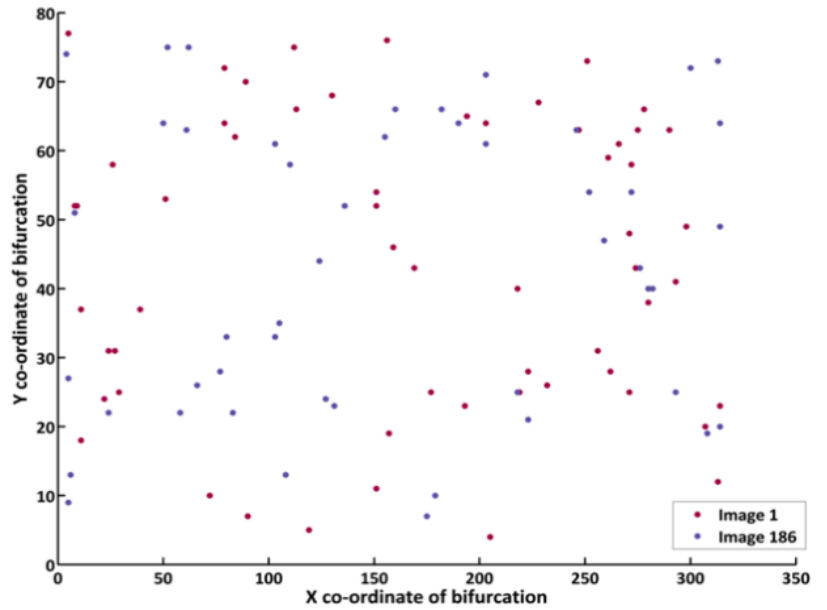

Figure 1.9: A scatter plot of the positions of the bifurcations of two images of different classes. The difference in positions of bifurcations is evident.

Note that the absolute positions ( $\mathrm{x}$ and $\mathrm{y}$ coordinates) of the bifurcations are not invariant to either rotation or translation. If we have two images of the same individual, but the subject rotated their finger when only one of the images was captured, the extracted bifurcations from one image would all be displaced relative to the bifurcations in the other. This can lead to the extracted features appearing to be very different, even though they are actually from the same subject. However, this problem was not encountered in this project because, as mentioned before, the vein-imaging device used to populate the SDUMLA-HMT database prevented subjects from rotating their fingers.

Nevertheless, in order to develop a robust classifier, it is important to bear in mind that we could form features which are invariant to rotation and translation by using relative distances and angles between bifurcations.

3) Clustering of Bifurcations: From Figure 1.8, it can be seen that another way to represent a set of bifurcations from multiple images of the same class is to group them into a set of $\mathrm{k}$ clusters. Each of these clusters would have a centroid position, and a list of these centroids would be the feature vector representative of an entire class.

With reference to the system architecture in Figure 3.1 , it is important to note that the data stored in the training database (list of centroids) is not of the 
same form as the feature vector (list of bifurcations) which is extracted and compared during identification.

We use the Support Vector Machine algorithm, to obtain the centroid positions of the clusters. An example of the clusters formed from five images of the same subject is shown in Figure 4.8. As multiple images of the same class will have almost identical bifurcations, a dense cluster

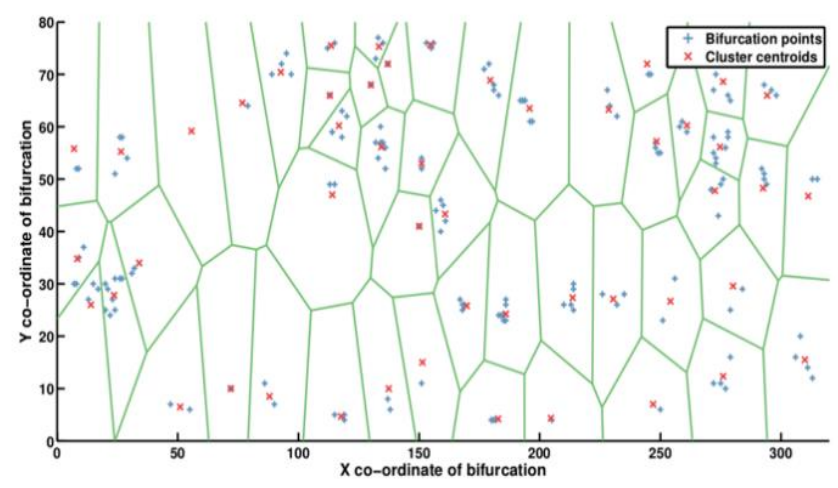

Figure 2.0: 50 Clusters formed from bifurcations of five of the six samples of one class. Most clusters contain at least five bifurcations - one from each sample. Note that there are a few cases where the centroid position and bifurcation are identical.

of similar points is formed. It can be seen in Figure 1.9 that most of the clusters have at least five bifurcations (since five images were used as input). Additionally, the effect of "outlier" bifurcation points is reduced since the bifurcations common to all the images would have a greater weight in determining the positioning of the cluster centroids. There are, however, a number of considerations we need to take into account when using the k-means algorithm.

4). Classification: The features, in the form of coordinates of bifurcations, are extracted from a query image and used to classify an individual. We propose a classifier: A classifier trained with a list of cluster centroids of all the bifurcation points from multiple images of the same class. An immediate issue is that the feature vector is of variable length. The list of $\mathrm{x}$ and $\mathrm{y}$ co-ordinates of the bifurcations have no natural ordering and each query image can have a different number of these points.
This issue still applies when training a classifier with a list of cluster centroids because the number of cluster centroids and the number of bifurcations extracted from the query image will not be equal. A well-known classifier such as a Neural Network or Nearest Neighbour is not appropriate. These classifiers expect ordered feature vectors of equal length and it does not make sense to pad short feature vectors with zeroes. The following example explains why:

Consider two feature matrices obtained from two images of the same subject as $v 1$ and $v 2$ respectively. The row of each matrix represents the $x$ and $y$ co-ordinates of each bifurcation. Due to segmentation errors in one of the images, assume that we obtain an extra bifurcation point for $v 2$, as shown by the example below:

$$
v_{1}=\left(\begin{array}{ll}
1 & 1 \\
3 & 3 \\
4 & 4
\end{array}\right) \quad v_{2}=\left(\begin{array}{cc}
1.1 & 1.1 \\
2 & 2 \\
3.1 & 3.1 \\
4.1 & 4.1
\end{array}\right)
$$

It is clear that both of these feature matrices are very similar. However, a "black-box" classifier cannot be blindly trained which assumes that features are ordered. In this example, the row $(3,3)$ of matrix $v 1$ will be compared to $(2,2)$ of $v 2$ and $(4,4)$ of matrix $v 1$ will be compared to $(3.1,3.1)$ of $v 2$. If we zero-pad $v 1$, then the row $(0,0)$ of $v 1$ will be compared to $(4.1,4.1)$ of matrix $v 2$.

The result is that the feature matrices will appear very different to the classifier even though they are clearly very similar. When classifying, we need to match appropriate rows of each matrix to one another.

The vein IR imaging implementation below follows up on the trained dataset detailed from the third chapter of this study. 


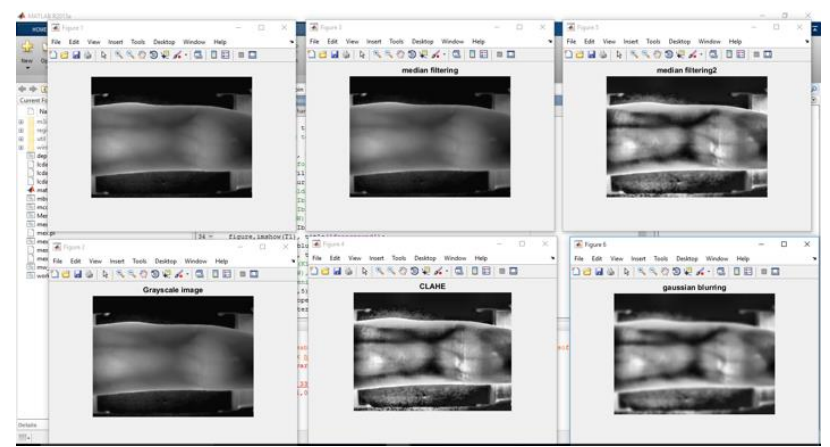

Figure 2.1: Output of enhanced vein IR finger image

As discussed already, in order to extract the intersection points of veins, there is a need to segment the veins from the rest of the image. A binarised image was produced in this stead, where the foreground (white) pixels represent the actual vein and the background (black) pixels represent the remaining tissues of the finger.

The extraction of the Region of Interest (ROI) and subsequent contrast enhancement using the CLAHE algorithm, which is basically contrast enhancement is relevant since features are easier to extract from images with greater contrast. Moreover, vein images tend to have non-uniform illumination and low contrast.

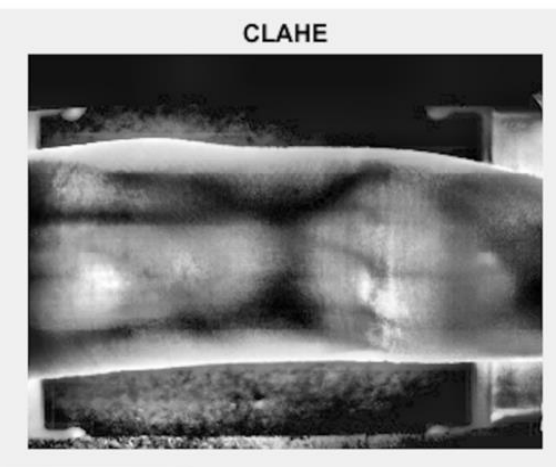

Figure 2.11: Improved contrast using the CLAHE algorithm

\section{Android Implementation}

The Android implementation follows up on our Matlab project in the previous section. This project uses an InfraRed (IR) imprint and checks the recorded imprint against the record in the fingerprint database to authorize or reject the transaction that is about to take place.

1) Product Dashboard Page: The product dashboard page is a single page which mimics an ecommerce dashboard; it includes a product, its description, and a purchase button which is where our biometric system gets integrated. The biometric function is used to authorize product purchase.

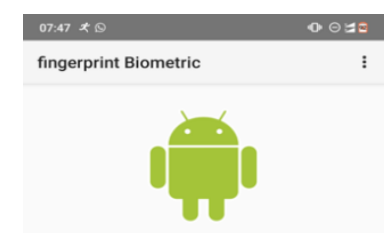

Droid Sticker Pack

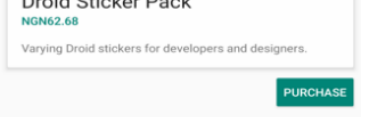

\section{Figure 2.12: Product Dashboard Page}

2) Fingerprint Settings Page: This page is where an individual user sets the option to use a fingerprint biometric to authenticate their transaction

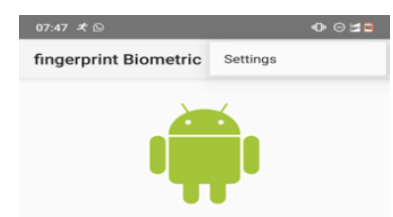

Droid Sticker Pack

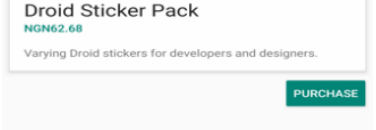

Figure 2.13: Fingerprint Settings Page

3) Fingerprint Prompt Page: This page pops up when the user selects the option to use their fingerprint for authentication. 


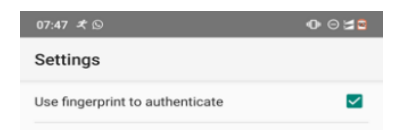

Figure 2.14: Fingerprint Prompt Page

4) Fingerprint Image Accept Page: As detailed above, if the fingerprint image is recognized, the system accepts and proceeds with the transaction, which is what happens in Figure 2.15 below.

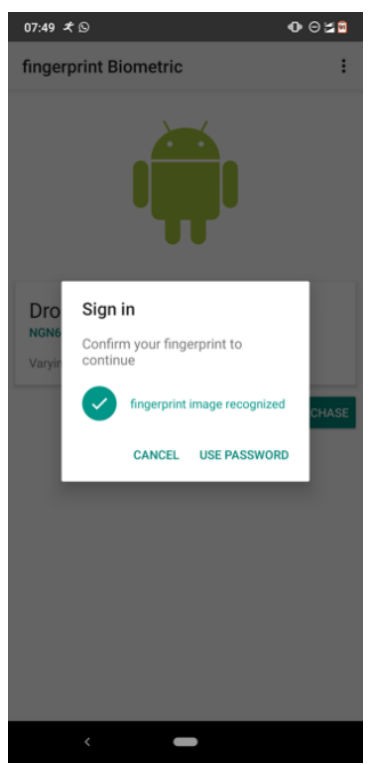

Figure 2.15: Fingerprint Image Accept Page

5) Fingerprint Token Generation Page: A token would get generated once a user is successfully authenticated. This token changes (meaning, a new token gets generated) each time a user gets authenticated to prevent spoofing. These tokens are generated using the SHA 256 algorithm.
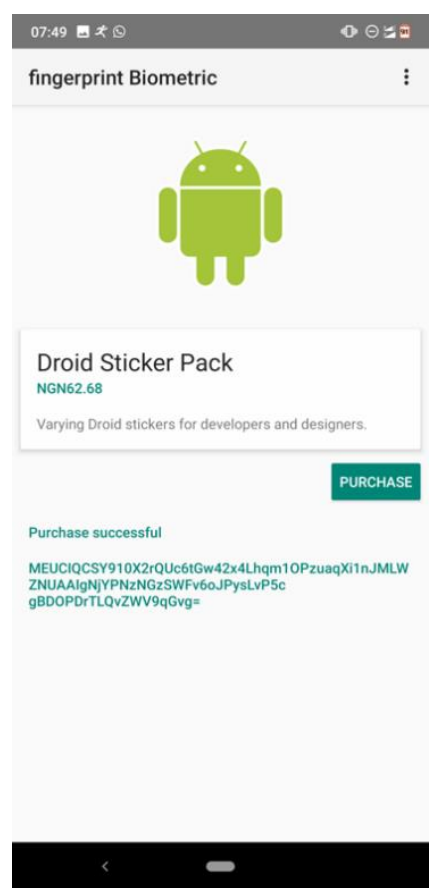

Figure 2.16: Fingerprint Token Generation Page

\section{E. Minutiae}

Minutiae can easily be extended from fingerprints to finger vein images as well. The segmented vein patterns from the sample image are analogous to ridges and also have bifurcations and terminations. Minutiae are features commonly used in fingerprint recognition. Minutiae refer to the various ways in which fingerprint ridges (the darker parts of a fingerprint image) can be discontinuous. Although there are several categories of minutiae, the most common are bifurcations - the point where one ridge divides into two - and terminations - the points where a ridge comes to an abrupt end. Below are fingerprint and finger vein minutiae shown in Figure 2.17.
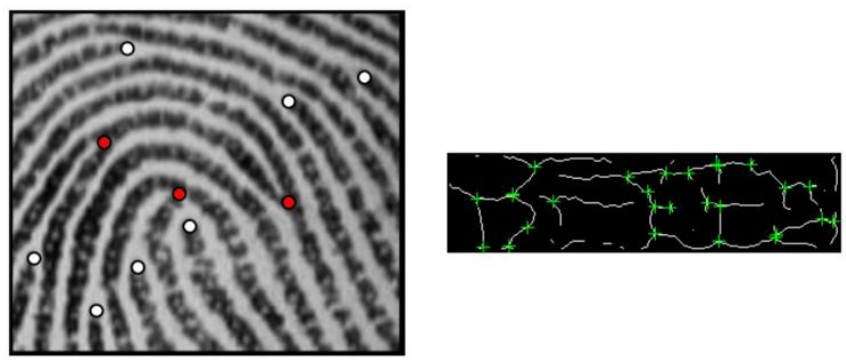

Figure 2.17: Minutiae obtained from fingerprints and finger vein images respectively

A number of researchers have extracted features from finger vein minutiae where the feature vector is a list of the $x$ and $y$ co-ordinates of the positions 
of the minutiae. However, such features are not invariant to rotations or translations.

\section{Conclusion}

In this paper, we designed a security system, using captured fingervein. Bifurcations have been extracted from the test fingervein as features and used a distinct classifier which uses these features to identify individuals. This classifier has tuneable parameters in addition to the threshold - the SVM classifier, which requires the value of $\mathrm{k}$, the number of clusters, to be optimised. Moreover, a modification has also been proposed to the SVM's distance metric.

An Android application which captures the fingerprint of an individual, reads it using a Matlab vein IR project, then processes the vein images using the trained Matlab project which includes feature extraction and classification has also been implemented and presented to indicate how a fingerprint biometric system works in contrast to fingervein systems.

\section{References}

[1.] David Mulyono, and Horng Shi Jinn, A Study of Finger Vein Biometric for Personal Identification, Proceedings of the IEEE International Symposium on Biometrics and Security Technologies (ISBAST 2008), pp. 1-8, 2008.

[2.] Wenming Yang, Qing Rao, and Qingmin Liao, Personal Identification for Single Sample using Finger Vein Location and Direction Coding, Proceedings of the IEEE International Conference on Hand-based Biometrics (ICHB), pp. 1-6, 17-18 March 2011.

[3.] Hua-Bin Wang, and Liang Tao, Novel Algorithm for Enhancement of Hand Vein Images based on Adaptive Filtering and Retinex Method, Proceedings of the IEEE International Conference on Information Science and Technology (ICIST), Wuhan, Hubei, China, pp. 857-860, 23-25 March 2012.

[4.] Shi Zhao, Yiding Wang, and Yunhong Wang, "Extracting Hand Vein Patterns from Low-Quality Images: A New Biometric Technique using Low-Cost Devices", Fourth International Conference on Image and Graphics (ICIG 2007), IEEE Computer Society, pp. 667-671, 22-24 Aug 2007.

[5.] Desong Wang, Jianping Li, and Gokhan Memik, "User Identification based on Finger-vein Patterns for Consumer Electronics Devices", IEEE Transactions on Consumer Electronics, Vol. 56, No. 2, pp. 799-804, May 2010.

[6.] Zhi Liu, and Shangling Song, "An Embedded Real- Time Finger-Vein Recognition System for Mobile Devices", IEEE Transactions on Consumer Electronics, Vol. 58, No. 2, pp. 522-527, May 2012.

[7.] Li Zhichao, Sun Dongmei, Liu Di, and Liu Hao, "Two Modality-Based Bi-Finger Vein Verification System", the 2010 IEEE 10 International Conference on Signal Processing (ICSP) pp. 1690-1693, 24-28 Oct 2010.

[8.] Lin Yang, Xiangbin Liu, and Zhicheng Liu, "A Skeleton Extracting Algorithm for Dorsal Hand Vein Pattern", 2010 International Conference on Computer Application and System Modeling (ICCASM 2010), pp. V13-92-V13-95, 2224 Oct 2010.

[9.] Jinfeng Yang, Jinli Yang, and Yihua Shi, "Finger-Vein Segmentation Based on Multichannel Even-symmetric Gabor Filters", IEEE International Conference on Intelligent Computing and Intelligent Systems 2009 (ICIS 2009), Vol. 4, pp. 500503, 20-22 Nov 2009.

[10.] Gongping Yang, Xiaoming $\mathrm{Xi}$, and Yilong Yin, "Finger Vein Recognition Based on a Personalized Best Bit Map", Journal on Sensors, Vol. 12, pp. 1738-1757, doi: 10.3390/s120201738, 2012.

[11.] Naoto Miura, Akio Nagasaka, and Takafumi Miyatake, "Feature Extraction of Fingervein Patterns based on Repeated Line Tracking and its Application to Personal Identification", Machine Vision and Applications, Vol. 15, pp. 194-203, 2004.

[12.] Lee H, S. H. Lee, T. Kim, and H. Bahn, "Secure User Identification for Consumer Electronics Devices", IEEE Transactions on 
Consumer Electronics, Vol. 54, No. 4, pp. 1798-1802, Nov. 2008.

[13.] Hashimoto J., "Finger Vein Authentication Technology and its Future", 2006 Symposium on VLSI Circuits, Digest of Technical Papers, pp. 5-8, 2006.

[14.] $\mathrm{Wu}$ J. D., and S. H. Ye, "Driver Identification using Finger-V ein Patterns with Random Transform and Neural Network", Expert System Applications, Vol. 36, pp. 5793-5799, 2009.

a. Jain, P. Flynn, and A. Ross, Handbook of Biometrics. Springer, 2007.

[15.] M. A. Olsen, D. Hartung, C. Busch, and R. Larsen, "Contrast enhancement and metrics for biometric vein pattern recognition," in Advanced Intelligent Computing Theories and Applications, pp. 425-434, Springer, 2010.

[16.] T. Y. Zhang and C. Y. Suen, "A fast parallel algorithm for thinning digital patterns," Commun. ACM, vol. 27, pp. 236-239, Mar. 1984.

[17.] R. Xiao, L. Zhang, and H.-J. Zhang, "Feature selection on combinations for efficient learning from images," in Proceedings of the Asian conference on computer vision, January, vol. 2, pp. 10741079, Citeseer, 2004. 